\title{
Antibubbles, liquid onions and bouncing droplets
}

\author{
N. Vandewalle, D. Terwagne, T. Gilet, H. Caps, S. Dorbolo* \\ GRASP, Institut de Physique, Université de Liège, 4000 Liège, Belgium
}

\section{A R T I C L E I N F O}

\section{Article history:}

Received 15 October 2008

Received in revised form 5 January 2009

Accepted 7 January 2009

Available online 17 January 2009

\section{Keywords:}

Droplets

Bubbles

Microfluidics

Encapsulation

Emulsion

\begin{abstract}
A B S T R A C T
In this paper, we emphasize our long series of experiments proving that the physical processes along fluid interfaces can be exploited for creating unusual fluidic objects. We report for the first time a couple of new fluidic objects so-called "liquid onions" and "mayonnaise" droplets. The study starts from the observation of antibubbles, exhibiting unstable liquid-air-liquid interfaces. We show that the lifetime of such a system has the same origin as floating/coalescing droplets on liquid surfaces. By analyzing such behaviours, we created droplets bouncing on a liquid bath. The methods and physical phenomena collected in this paper provide a basis for the development of a discrete microfluidics. Open questions are underlined, experimental challenges and future applications are proposed.
\end{abstract}

(c) 2009 Elsevier B.V. All rights reserved.

\section{Introduction}

The main subject of this paper is to collect our major results obtained from a series of experimental works on droplet-based fluidic objects [1-9]. The paper gives an overview of what was done in the past 5 years in the Group for Research and Applications in Statistical Physics (GRASP) at the University of Liège, Belgium. Nevertheless, the present paper aims to overcome the state-of-the-art review by emphasizing various open fundamental questions and by proposing new experiments. Moreover, two new bifluidic objects will be reported for the first time: liquid onions and mayonnaise droplets.

The present story starts in 2003 with a systematic study [1] of antibubbles which raised a lot of questions. Some of them are still open nowadays. In particular, a deep analysis for the stability of antibubbles provided key mechanisms for understanding the physics behind numerous droplet-based systems. The next section will be focused on the case of antibubbles. Since that study, two parallel approaches have been developed: (i) the creation of fluidic objects similar to antibubbles, floating below the air-liquid interface; (ii) the interaction of fluidic objects and a liquid-air interface. The first approach tends to create complex emulsions or giant vesicles, with eventually the use of more than two liquid components. The second approach leads to different ways of manipulating droplets without any contact with a solid object. The later creates a basis for lots of potential applications in discrete microfluidics.

\footnotetext{
* Corresponding author. Tel.: +32 43663703; fax: +32 43663629.

E-mail address: nvandewalle@ulg.ac.be (N. Vandewalle).
}

The Fig. 1 summarizes all the fluidic systems which will be discussed in the present paper: antibubbles, encapsulated droplets, floating/coalescing droplets, bouncing droplets, so-called "mayonnaise" droplets.

\section{Antibubbles}

Antibubbles were first reported in 1932 by Hugues and Hugues [10]. They were coined "antibubbles" in the 1970s [11]. Only a few works $[1-3,12-14]$ have been devoted to a quantitative study of antibubbles. They are mainly focussed on either the formation of antibubbles or the stability of such objects.

Antibubbles are created when a soapy liquid jet is impacting a liquid bath of the same liquid. Fig. 2 (top-left) presents two typical pictures of the impacting jet leading to the formation of antibubbles. The liquid jet crosses through the interface and a thin air film is entrained below the interface. The cylindrical air film eventually decomposes into antibubbles. Very recently, it has been found [13] that only a narrow set of parameters (like liquid viscosity and liquid velocity) allows the formation of antibubbles since two mechanisms should coincide: lubrication process for air entrapment and pinching of the jet below the surface. Air pressure is also a relevant parameter, that has been clearly identified in Ref. [2].

As observed in Fig. 2(top-right), antibubbles look like spherical air bubbles but they exhibit a dark and thick border when lighted by the rear. The amount of air trapped in an antibubble is quite small. If a uniform air film is considered, the thickness $\varepsilon$ should be around $3-5 \mu \mathrm{m}$ [1]. The dark and thick border is due to total reflection of light. The typical diameters of antibubbles range from a few millimeters up to $3 \mathrm{~cm}$. Larger fluid entities are not observed. 


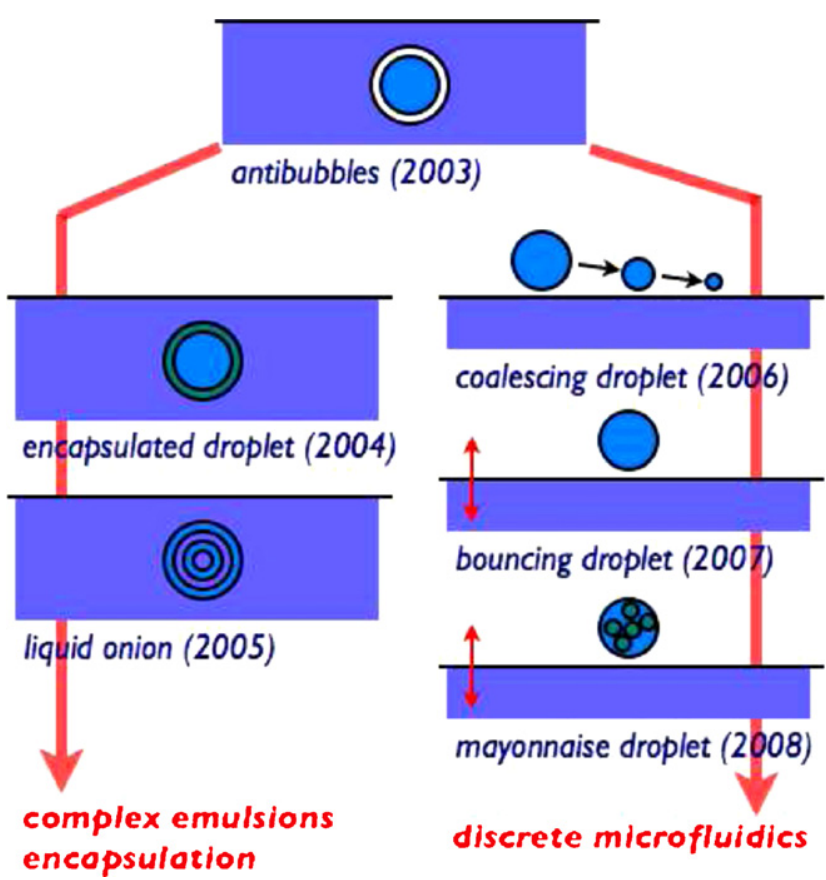

Fig. 1. Sketch of all fluidic objects considered in this paper. Interfaces are emphasized by dark curves. The colour indicates the presence of liquid: either water or silicon oil. Water may be also mixed with surfactant molecules. The white color is for the air. Arrows indicate the approaches that have been developed. (For interpretation of the references to color in this figure legend, the reader is referred to the web version of the article.)

From the point of view of the interface structure, antibubbles cannot be stable. Indeed, the surfactant molecules forming layers along the air film present hydrophobic parts face to face, as shown in Fig. 2(bottom-left). By opposition to a common soap film, no repulsive interaction exists in order to stabilize the air film; only attractive forces act. Although the absence of such a stabilization mechanism, an antibubble can last a few minutes before breaking. Indeed, the average lifetime of an antibubble is around $100 \mathrm{~s}$, while a few of them could last as long as $10 \mathrm{~min}$. In order to explain the apparent stability of antibubbles, we proposed [3] a simple model based on the drainage of air in the spherical shell from the bottom to the top. Indeed, we observed that the top of an antibubble presents a small bump meaning that air is accumulating there. By assuming a viscous drainage of air from the south pole to the north pole of an antibubble of radius $R$, we derived [3] a characteristic time:

$\tau=\frac{3 R \eta}{\rho g \varepsilon_{c}^{2}}$,

where $\varepsilon_{c}$ is the critical distance between surfactant layers, below which the air film breaks. Using $\varepsilon_{c} \approx 100 \mathrm{~nm}$ which is the typical interaction range for the van der Waals forces, one obtains roughly $\tau \approx 3600 \mathrm{~s}$, in agreement with our observations. However, the model does not take into account heterogeneous drainage due to local thinning of the air film. This explains also why large antibubbles are not created since the air entrapment depends on the antibubble size $R$. It has been measured [14] that $\varepsilon \sim R^{-1}$. As a consequence, the air film thickness of large antibubbles is close to the critical distance between layers $\left(\varepsilon \approx \varepsilon_{c}\right)$. This corroborates the experimental observation that large antibubbles are not created/reported.

Open questions are still numerous. The role played by the surfactant molecules is still unclear. We know that different amphiphilic molecules leads to significative differences in the antibubble lifetimes. It would be a real challenge to investigate particular molecules which decrease the air film drainage rate, and thus increase the antibubble lifetime. Another interesting point is the apparent stability of antibubbles submitted to large deformations (large shear rates). For example, Fig. 2(bottom-right) presents a picture of a stretched antibubble around a Rankine vortex created when the liquid bath is rotated. Deformations up to 10-20 antibubble diameters have been observed.

From the point of view of applications, antibubbles provide a simple mean to create micro-sized bubbles. Indeed, the air film is extremely thin when the antibubble breaks. The antibubble breaking results in the creation of a high number of tiny air pockets (a few microns in diameter) in addition to the rising air bubble resulting from the north pole cap.

\section{Encapsulation and liquid onion}

In order to increase the lifetime of antibubble-like objects, one can replace air by a viscous fluid like silicon oil. The idea is to generate macroscopic Water in Oil in Water (WOW) droplet [15]. Since antibubbles are created from an impacting jet on air-water interfaces, WOW droplets are obtained by impacting droplet on a oil-water interface [9], also reported by Galvin et al. [16]. The encapsulation of the incoming droplet with a thin oil layer can be observed on Fig. 3 (left). Encapsulation needs high velocities at the impact. Indeed, the interaction time $t_{i}$ should be smaller than the capillary time $t_{c}$, being roughly $1 \mathrm{~ms}$. The condition reads:

$t_{i}=\frac{e}{v}<\sqrt{\frac{\eta^{2}}{\rho g \gamma}}=t_{c}$.

Fig. 3(right) presents such an object near the interface. The diameter of the encapsulated droplet is roughly $1 \mathrm{~mm}$. No surfactant molecule is required to create such particular object! However, the latter is unstable with a lifetime controlled by the viscosity of oil (see Eq. (1)).

For high impact velocities and for particular sets of parameters, one observes eventually a multi-layered droplet as shown in Fig. 3(right). Such a fluidic object is characterized by two spherical oil shells. Starting from the center, we observe water coming from the bath, an oil shell, a water shell coming from the impacting water droplet, a second oil shell and finally the water bath. This has been evidenced by colouring differently the liquid phases. In fact, multilayers originate from a particular deformation (invagination) of the oil-bath interface which sucks and traps a small amount of bath liquid. Such an object is called a "liquid onion" and its lifetime is limited by the coalescence of the successive layers with the corresponding phases.

Open questions concern the maximum number of layers that can be encapsulated in such systems. We obtained droplets with two successive oil layers. Can we produce more complicated fluidic systems? A part of that question is connected to the last section of the present paper since we created droplets containing dozen of microdroplets. Another question concerns the possibility to form three phases droplets by using three non-miscible liquids A-B-C. Preliminary experiments prove that triple phase droplets could exist, nevertheless such an experimental study raises new difficulties related to the variety of interfaces created in a small object.

By replacing oil by a photopolymer, one could solidify the spherical shell around the liquid droplet. This has been successfully achieved in our laboratory for a demonstration. Such an encapsulation provides an original way to ensure the protection of delicate liquids. In so doing, a lot of application can be found.

\section{Floating and coalescing droplet}

The dynamics of the air film squeezed between two liquid phases is rather interesting. For example, in the Fig. 2, one can observe 

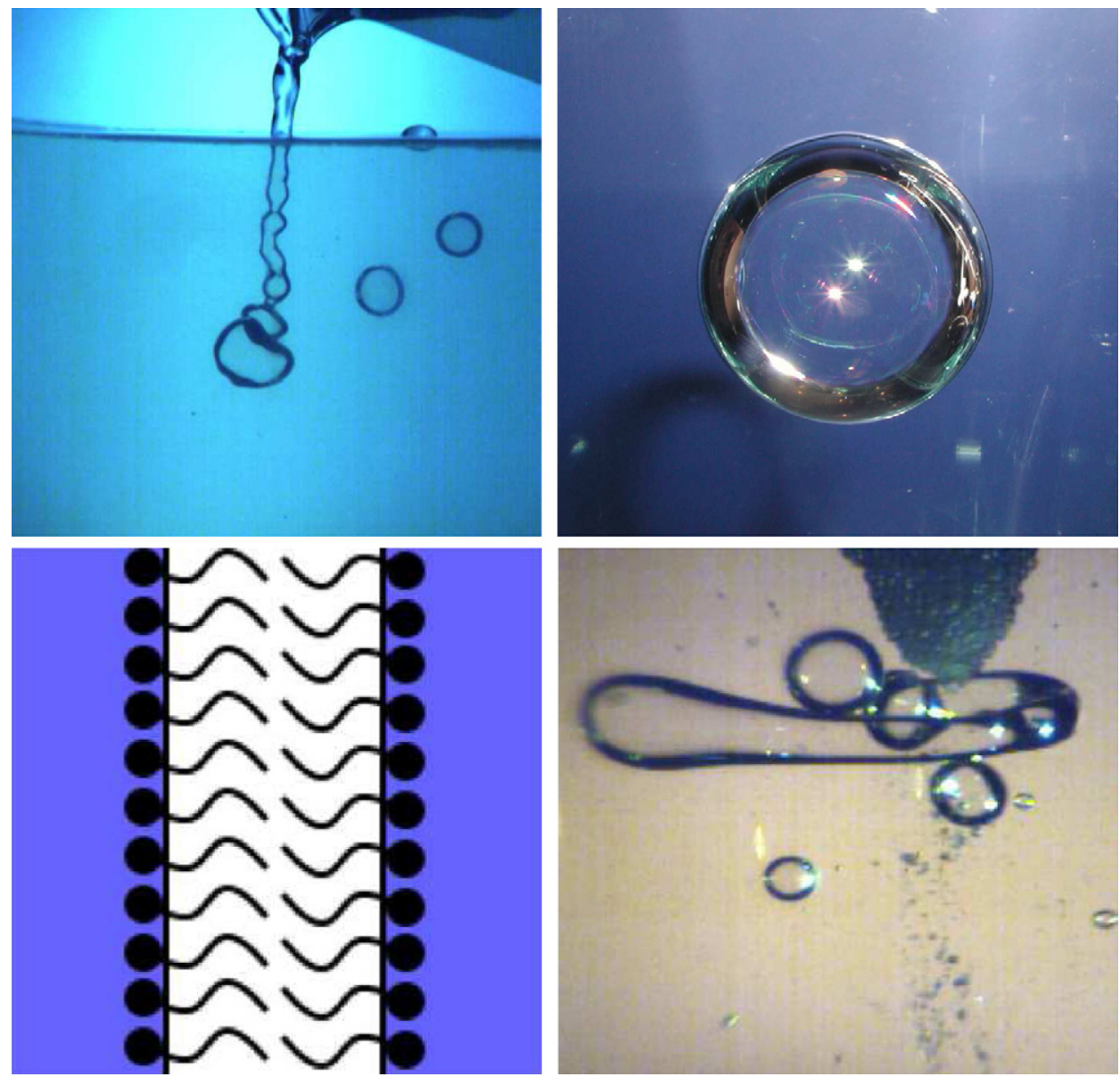

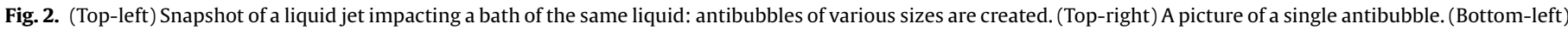

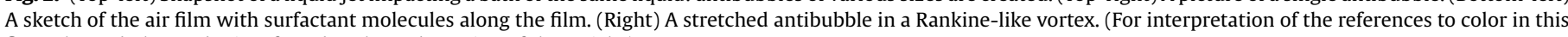
figure legend, the reader is referred to the web version of the article.)

a droplet floating along the air/liquid interface. This droplet (produced by the impacting jet) remains an instant (typically a fraction of a second) on the interface before coalescing. Using the model proposed in Eq. (1), one obtains $\tau=1 \mathrm{~s}$ for a millimetric droplet. This implies that the air drainage delays the coalescence (in the next section, this coalescence will be prevented by renewing the air film). Such a delay to coalescence has been studied since the 1960s [17]. It has been reported that the coalescence could be either partial or total. In most cases, a total coalescence occurs when the air film breaks. However, depending on liquid viscosities and surface tension, the coalescence may be partial. It remains a small daughter droplet lying on the surface. In fact, nearly $90 \%$ of the initial volume can be lost during the coalescence event [18]. Fig. 4 presents both situations.

In the case of a partial coalescence, the daughter droplet could also remain an instant on the liquid bath before partially coalescing.

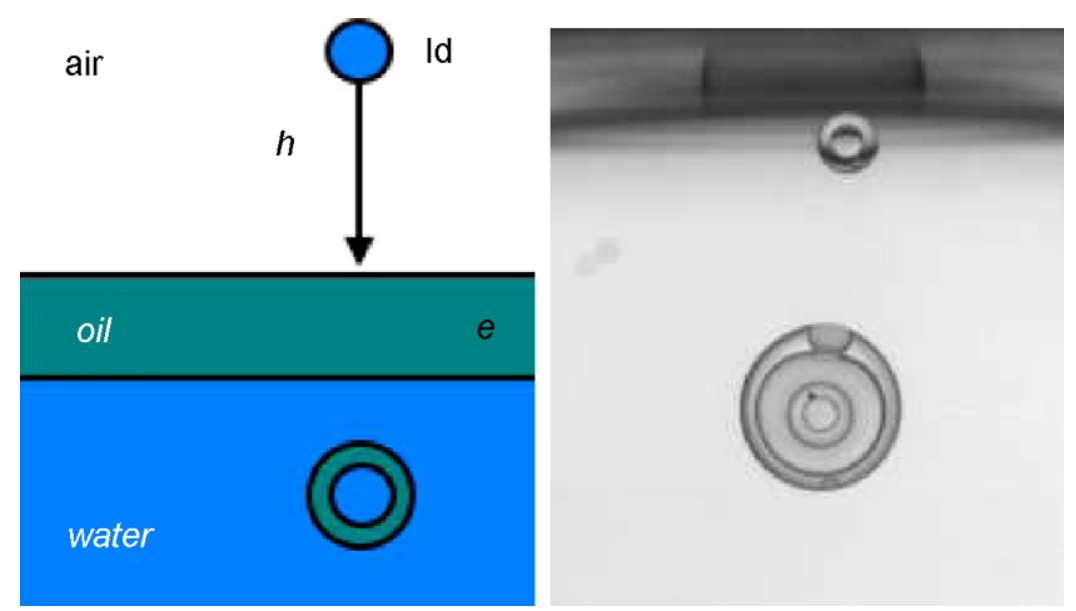

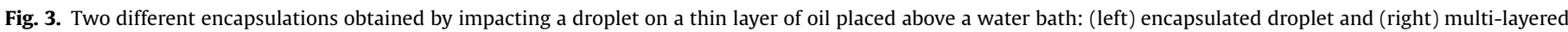
fluid droplet, called "liquid onion". 

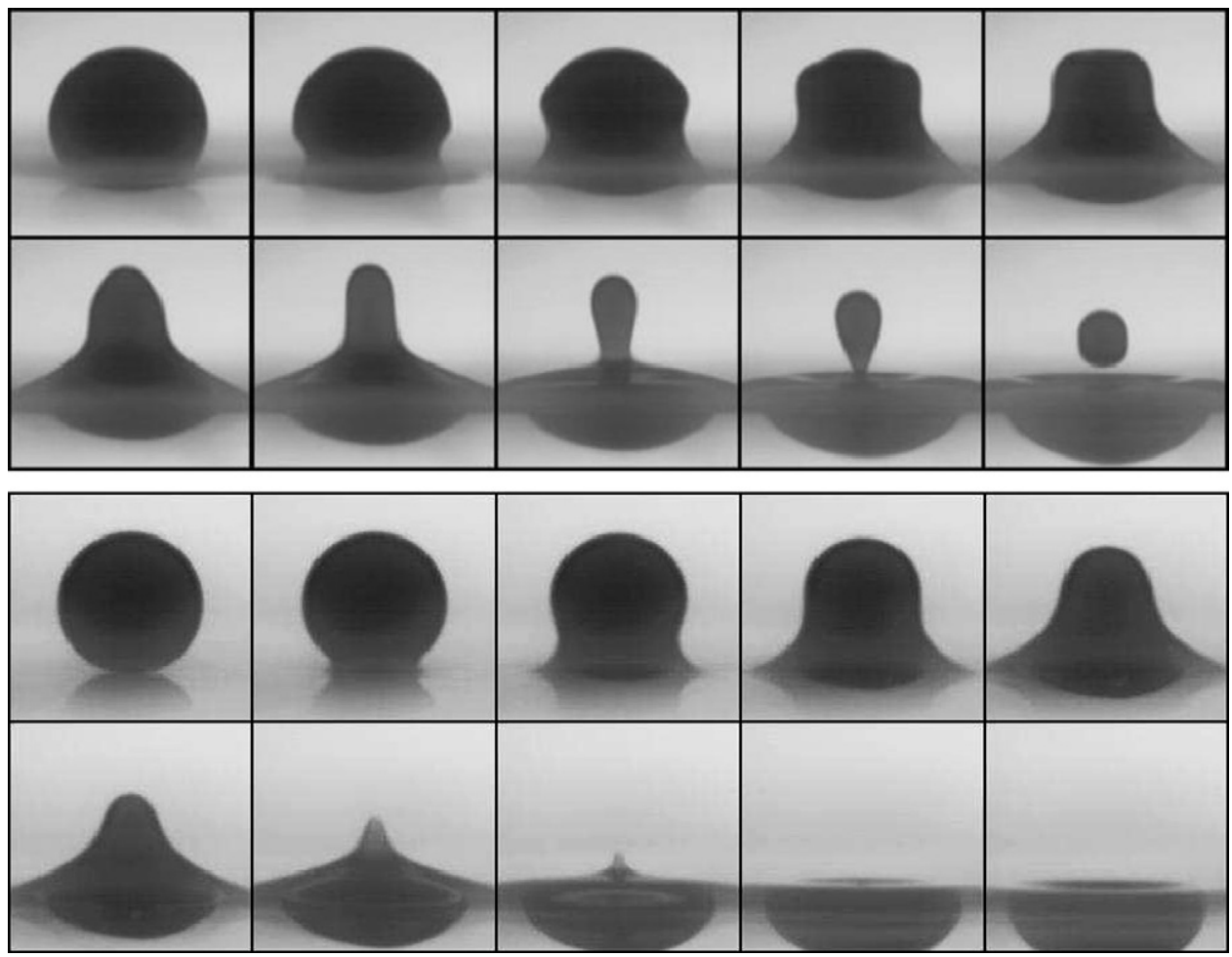

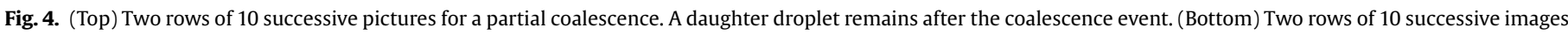
for a total coalescence.

This process could be repeated a given number of times. Up to seven successive events have been observed in our experiments using two immiscible fluids (water/oil). Tiny droplets down to $20 \mu \mathrm{m}$ have been observed at the end of this cascade of events. As proposed by Blanchette and Bigoni [19], the partial coalescence is due to capillary waves initiated when the droplet starts to interact with the liquid bath. Those waves propagate towards the north pole of the spherical cap. There, the addition of such waves could lead to a pinch off and thereafter to a daughter droplet detachment. One understands that the ratio between viscous and capillary forces should be low in order to avoid the damping of capillary waves. The relevance of critical Onhesorge numbers:

$O h=v \sqrt{\frac{\rho}{\gamma R}}$

has been evidenced [4]. Our work predicts a maximum of 11 successive coalescence events for the mercury/acetone system [4]. This has not yet been observed and represents a real challenge for future experimental works.

Open questions concern the effect of surfactants when the water droplets are coalescing on a water/surfactant bath. Indeed, the relaxation times associated to surfactant mobility and coalescence have similar values, leading to additional phenomena such as microscopic jet formation and droplet horizontal motion. Such experimental observations have not yet found a physical interpretation.

The cascade of droplet coalescences could lead to applications for microdroplet delivery. Indeed, microdroplets as small as $20 \mu \mathrm{m}$ could be produced in a controlled way!

\section{Bouncing droplet}

For avoiding the coalescence of a droplet, the drainage rate of the air film have to be strongly reduced. A simple and elegant method to achieve that purpose is to inject fresh air below the droplet by oscillating the interface. Couder et al. [20] proposed to place a viscous oil droplet on a vibrated bath of the same oil. Depending on the size of the droplet, the reduced acceleration $\Gamma=A \omega^{2} / g$ must be larger than a threshold for bouncing the droplet forever. Below this threshold $\Gamma_{\text {th }}$, the droplet coalesces. Based on lubrication of the air film, a model gives a threshold scaling like

$\Gamma_{\text {th }}=1+\beta \omega^{2}$,

where the constant $\beta$ collects droplet size and viscous effects. This model assumes that the maximum acceleration $A \omega^{2}$ of the liquid bath has to be always larger than $g$, i.e. $\Gamma>1$. The experiments are usually conducted with frequencies around $50 \mathrm{~Hz}$.

In order to decrease the number of parameters, a low viscous droplet has been placed on a vertically vibrated high viscous bath. The role of the bath can be then neglected. In that case, the situation is completely different since the threshold $\Gamma_{\text {th }}$ could be significantly below 1 [7]. Moreover, minima are observed in the curve giving the acceleration threshold with respect to the frequency. The system droplet-air film can resonate! In fact, the droplet-bath system exhibits Rayleigh resonance modes for which droplet shapes are roughly spherical harmonics $Y_{\ell}^{m}$ [21]. Fig. 5 (top) presents in a row the successive images of a resonant mode $\ell=2, m=0$. More interestingly, the $m \neq 0$ modes lead to self-propelling droplets due to asymmetrical oscillations of the interface along the horizontal direction. Pictures of such rolling droplet are shown in Fig. 5(bottom). It should be also noted that chaotic motion has also been recently reported [22].

Open fundamental questions concern the resonant modes of bouncing droplets. How to control them? Is it possible to observe transitions from a mode to another one? What are the velocities of self-propelling droplets? Applications of bouncing droplets are numerous since it is possible to manipulate a droplet without touching it! 


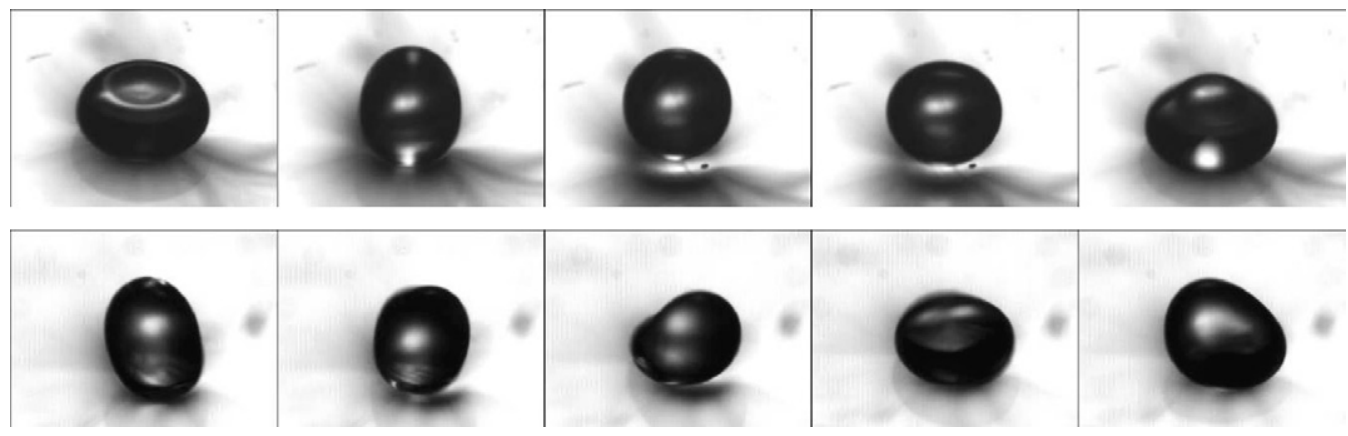

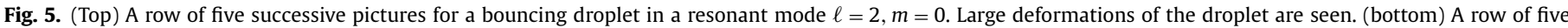
successive images for a self-propelling droplet (so-called rolling droplet) in a resonant mode $m \neq 0$.
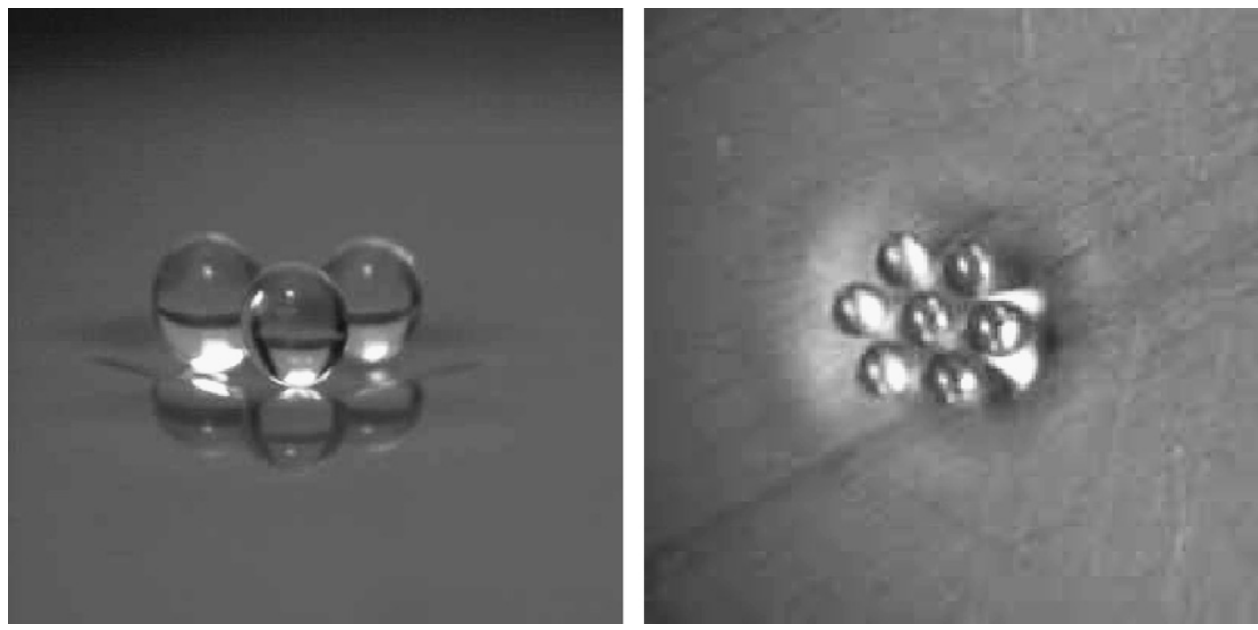

Fig. 6. Rafts of a set of droplets. (Left) Three droplets bounce synchronously. (Right) Seven droplets form a regular crystal network.

\section{Interacting droplets}

When two or more droplets are placed on a vibrating interface, complex attractions and repulsions are observed [23], leading to ordered structures such as crystal-like rafts [24]. Such structures are illustrated in the Fig. 6. Hexagonal structures are seen. Depending on the frequency/viscosity parameters, the stable interdistance between neighboring droplets can be modified. Some rotational or horizontal motion of droplet rafts have been also reported [8]. They are observed when droplets have different sizes.

It is also possible to induce droplet mixing and collisions onto the vibrated interface as suggested in our recent work [5]. In that case, the parameters are chosen in order to bounce small droplets while large ones are unstable. Collisions between small and large droplet induce coalescence of large droplets only. This results in a size selection mechanism.

\section{Mayonnaise droplet}

A bouncing oil droplet has initially a liquid core made of a water/surfactant mixture surrounded by oil. When the viscosity of the oil is low and when the acceleration of the liquid bath is high, the droplet is highly deformed at each cycle. Such deformations induce the introduction of tiny oil droplets into the water core of the bi-droplet. The number of inclusive droplet is limited when the volume of the microdroplets becomes comparable to the volume of the core. After a few cycles, the oil droplet contains a set of water microdroplets. A microemulsion is thus contained in the bouncing droplet. In Fig. 7, a snapshot of a mayonnaise droplet is presented.

Open questions concern the control of the emulsion inside the droplet as a function of the vibration characteristics $(A, \omega)$ and liquid properties $(\gamma, \eta)$. This study is currently performed by one of the authors. Similarity to foam systems [25], the stability of the

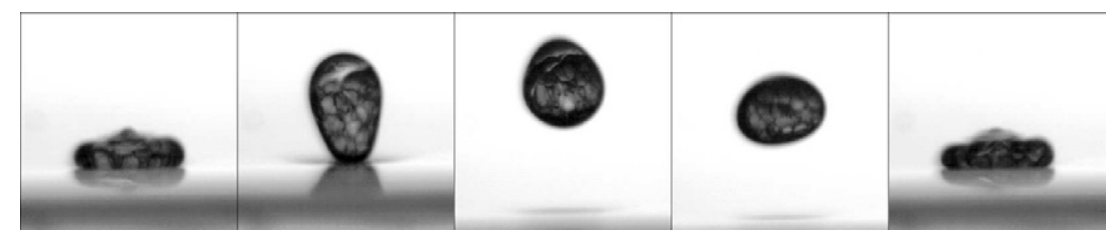

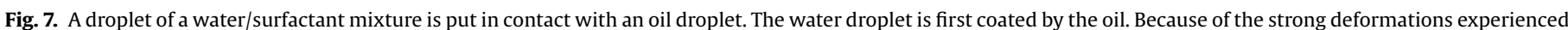
by this resulting bifluidic system, small oil drop are created and are floating in the water/surfactant core of the "mayonnaise droplet". 
created emulsion should be also studied. Applications can be envisaged from encapsulation of precious liquid to controlled reactions in droplets.

\section{Conclusion and perspectives}

The manipulation of droplets remains a big issue regarding any microfluidics application. Very nice and reproducible devices do exist and are commercialized. The originality of the presented experiments resides in the simplicity of the manipulation. Using the energy of an impact, is sufficient to generate complex multilayered droplet in a reproducible way. However, the underlying physics of the dynamical processes remain mysterious and the main ingredients are sometimes still to be discovered.

One can easily imagine various applications in food industry and pharmaceutics. The bouncing of droplets provides an elegant way to manipulate (mixing, moving, storing) droplets for discrete microfluidic perspectives. The number of exotic fluidic objects that one can create is only limited by our imagination.

\section{Acknowledgements}

NV thanks the organizers of Eufoam 2008 for the invitation. SD/TG would like to thank FNRS/FRIA for financial support. The Authors would like to thank COST Action P21 Physics of droplets (ESF) for financial help.

\section{References}

[1] S. Dorbolo, H. Caps, N. Vandewalle, New J. Phys. 5 (2003) 161.

[2] S. Dorbolo, N. Vandewalle, cond-mat/0305126 (2003).

[3] S. Dorbolo, E. Reyssat, N. Vandewalle, D. Quéré, EuroPhys. Lett. 69 (2005) 966.

[4] T. Gilet, K. Mulleners, J.P. Lecomte, N. Vandewalle, S. Dorbolo, Phys. Rev. E 75 (2007) 036303

[5] T. Gilet, N. Vandewalle, S. Dorbolo, Phys. Rev. E 76 (2007) 035302(R).

[6] D. Terwagne, N. Vandewalle, S. Dorbolo, Phys. Rev. E 76 (2007) 056311

[7] T. Gilet, D. Terwagne, N. Vandewalle, S. Dorbolo, Phys. Rev. Lett. 100 (2008) 167802.

[8] A. Eddi, D. Terwagne, E. Fort, Y. Couder, EuroPhys. Lett. 82 (2008) 44001

[9] S. Dorbolo, N. Vandewalle, D. Quéré, in preparation.

[10] W. Hughes, A.R. Hughes, Nature 129 (1932) 59.

[11] C.L. Stong, Sci. Am. 230 (1974) 116.

[12] A. Tufaile, J.C. Sartorelli, Phys. Rev. E 66 (2002) 056204.

[13] P.G. Kim, H.A. Stone, EuroPhys. Lett. 83 (2008) 54001.

[14] P.G. Kim, J. Vogel, Colloids Surf. A 289 (2006) 237.

[15] J. Hanson, C. Chang, S. Graves, Z. Li, T. Mason, T. Deming, Nature 455 (2008) 85.

[16] K.P. Galvin, S.J. Pratten, S.R. Biggs, G.M. Evans, Langmuir 22 (2006) 522.

[17] G.E. Charles, S.G. Mason, J. Colloid Sci. 15 (1960) 105.

[18] S.T. Thoroddsen, K. Takehara, Phys. Fluids 12 (2000) 1265.

[19] F. Blanchette, T.P. Bigioni, Nat. Phys. 2 (2006) 254.

[20] Y. Couder, E. Fort, A. Boudaoud, C.H. Gautier, Phys. Rev. Lett. 94 (2005) 177801.

[21] S. Dorbolo, D. Terwagne, N. Vandewalle, T. Gilet, New J. Phys. 10 (2008) 113021.

[22] T. Gilet, J.W.M. Bush, Phys. Rev. Lett. 102 (2009) 014501.

[23] S. Protière, S. Bohn, Y. Couder, Phys. Rev. E 78 (2008) 036204.

[24] S.I. Lieber, M.C. Hendershott, A. Pattanaporkratana, J.E. Maclennan, Phys. Rev. E 75 (2007) 056308.

[25] N. Vandewalle, J.F. Lentz, S. Dorbolo, F. Brisbois, Phys. Rev. Lett. 86 (2001) 179. 